\title{
Kinematic Analysis of Three-wire-driven Parallel (TWDP) Robot
}

\author{
Tao $\mathrm{Yu}^{1,}$, , Qingkai Han* 2 \\ ${ }^{1}$ School of Electromechanical Automobile Engineering, Yantai University, Yantai 264005,China \\ ${ }^{2}$ School of Mechanical Engineering, Northeastern University, Shenyang 110004, China \\ ayt_126@126.com * qhan@mail.neu.edu.cn (Corresponding author)
}

Keywords: Wire-driven, Parallel robot, Kinematics, Statics

\begin{abstract}
In the paper, a novel new gravity-constrained (GC) three-wire-driven (TWD) parallel robot is proposed. With its mechanism model, three typical kinematics analytical models, including horizontal up-down motion, pitching motion and heeling motion and their corresponding simulations are given in detail. In static analysis, the change of tensions in the wires is calculated based on previous kinematics analysis. The simulation results show the robot has good movement stability. The paper can provide useful materials to study of dynamics and control on wire-driven robot.
\end{abstract}

\section{Introduction}

The wire-driven mechanism, a kind of mechanism using wire or tendon to transform power and motion, has advantages of lighter mass, lower price and more widely workspace than that driven by hydraulic cylinder or lead screw. So more and more wire-driven mechanism or robot are designed and studied [1-3]. In 1995, a new high speed parallel robot named "FALCON-7"[4] driven by tensioned wire with rigidity was firstly reported by S. Kawamura on the international conference of robot and automation, which attracted widely attention of international scholar. After that, some new kinds of wire-driven parallel robots are proposed, such as "NIST ROBOCRANE" by J. Albus [5] and "CABLEV" [6] by Thomas Maier, etc.

In the paper, with its mechanism model, three typical kinematic analytical models, including horizontal up-down motion, pitching motion and heeling motion and their corresponding simulations are given in detail.

\section{Construction of the Robot and Degree of Freedom Analysis}

Construction of the Robot. The robot consists of base, platform (manipulator), pulley, pillar, wire and motor, as shown in Fig. 1. Through adjusting of the length of the wire, the position and attitude of the platform can be controlled.

The platform's shape is isosceles triangular. Each corner of the platform is connected with one end of a strip of wire, and the other end is wrapped around the shaft of the motor through the big pulley on the top the pillar and the small pulley at the bottom of the pillar consequently. By the gravity of platform itself, the wires are tensioned. Thus, a novel gravity-constrained (GC) three-wire-driven (TWD) parallel robot is proposed.

Freedom of the Robot. From Fig. 1, the mechanism of the robot can be obtained as shown in Fig.2, in which $O X Y Z$ is global coordinate and $o x y z$ is local coordinate of platform. Each strip of the wire can be viewed as a combination of a shifting pares and a revolute pare. At three top ends of the pillars and the three corners of the platform, the wires can be regarded as six Hooke pares. When the length of the wire, that is $l_{1}, l_{2}$ and $l_{3}$ respectively, is determined, the position and attitude of platform can be determined. From the analysis of the Fig.3, the platform can move translational along the $\mathrm{Z}$ axis, called up-down movement, and rotate around $\mathrm{X}$ and $\mathrm{Y}$ axis, called heeling and pitching movement respectively. Thus, the robot can be treated as a three freedom system. 


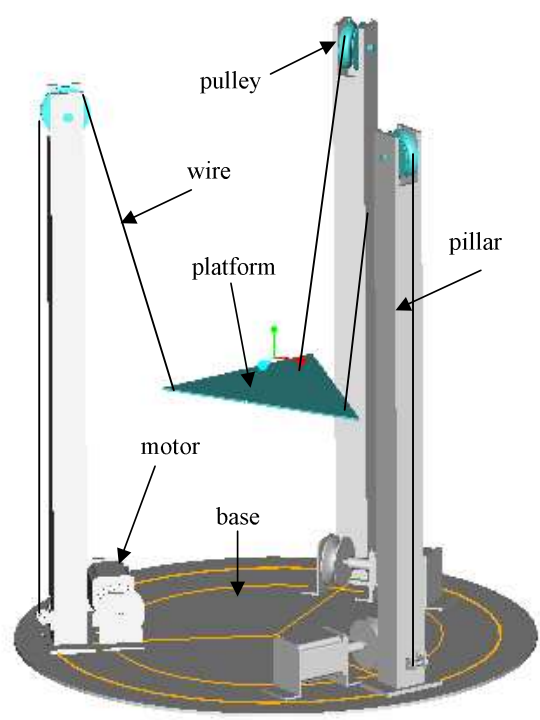

Fig. 1. Structure of robot

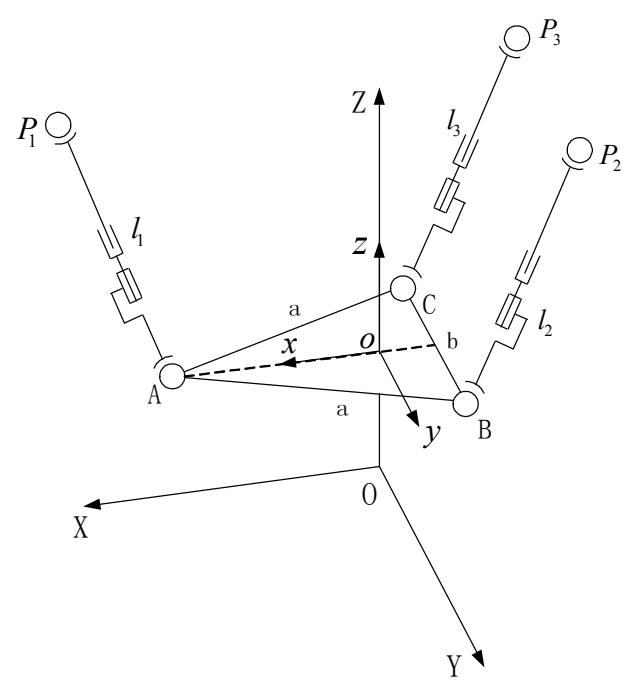

Fig. 2. Machanism of the robot

\section{Kinematic Analysis and Simulation}

Three typical motions, e.g. horizontal up-down motion, pitching motion and heeling motion are discussed in this part.

Horizontal up-down motion. In this motion, the platform moves symmetrically with $X$ axis. The spatial problem can be transferred as a planar problem, as shown in Fig.3 (a) (b).

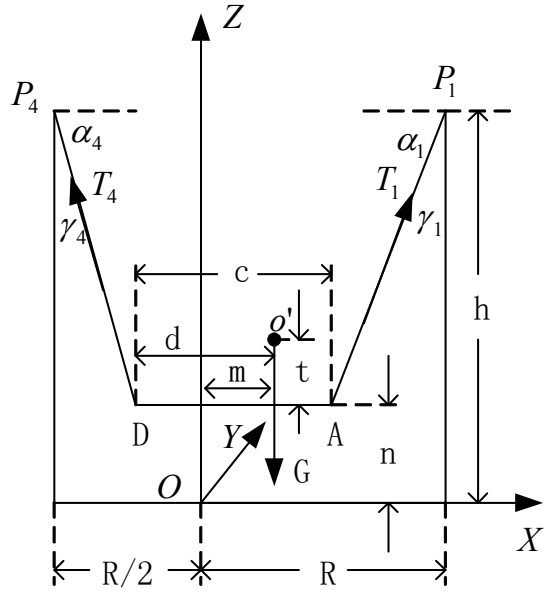

(a) side view

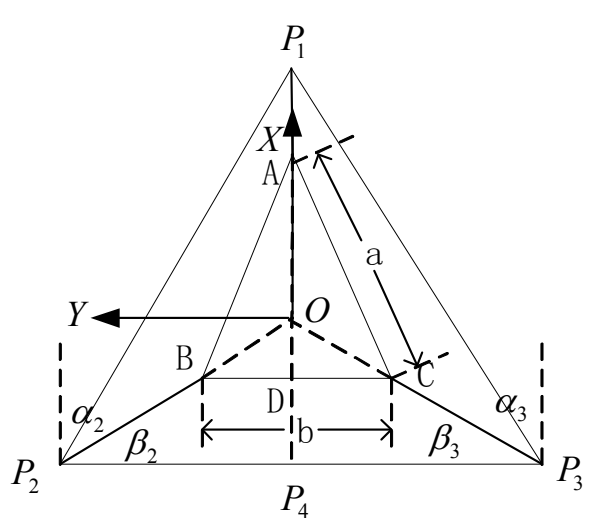

(b) top view

Fig. 3. The horizontal up-down situation of platform

The structural parameters of the robot are, $t=0 \mathrm{~mm}, R=247.5 \mathrm{~mm}, b=250 \mathrm{~mm}, d=91 \mathrm{~mm}$, $c=273 \mathrm{~mm}, h=750 \mathrm{~mm}$, and $G=2 \mathrm{~N}$, where $t$ is the vertical distance from the center of gravity of loaded platform to the base. $R$ is the radius of circle where the pillars locate. $b$ is the length of base of isosceles triangle platform. $d$ is the distance from $o$ ' to platform. $c$ is the distance from the top of platform to the base edge. $h$ is the height of pillar. $G$ is the total gravity of platform and loads. The tensions in the wire $\mathrm{P}_{1} \mathrm{~A}, \mathrm{P}_{2} \mathrm{~B}$ and $\mathrm{P}_{3} \mathrm{C}$ are identified as $T_{1}, T_{2}$ and $T_{3}$. A piece of virtual wire $\mathrm{P}_{4} \mathrm{D}$ is introduced for the convenience of analysis, the endpoint $\mathrm{P}_{4}$ and $\mathrm{D}$ are located as the middle of $\mathrm{P}_{2} \mathrm{P}_{3}$ and $\mathrm{BC}$. In general case, the platform together with loads are considered as a whole body, which is called manipulating target. The centre-of-gravity position is $o^{\prime}$, as shown in Fig.3(a).

By using force balancing, torque balancing and geometrical relationship of robot structure, the following equations can be established in the global coordinates. 


$$
\left\{\begin{array}{l}
l_{1} \cos \alpha_{1}+c+l_{4} \cos \alpha_{4}=3 R / 2 \\
l_{1} \cos \gamma_{1}=l_{4} \cos \gamma_{4} \\
T_{1} \cos \gamma_{1}+T_{4} \cos \gamma_{4}=G \\
T_{1} \cos \alpha_{1}=T_{4} \cos \alpha_{4} \\
T_{4} \cos \alpha_{4} \cdot n-T_{4} \cos \gamma_{4}(d-m)+T_{1} \cos \gamma_{1} \\
(m+c-d)-T_{1} \cos \alpha_{1} \cdot n-G \cdot m=0 \\
l_{1} \cos \gamma_{1}+n=h
\end{array} .\right.
$$

where, $\alpha_{i}, \beta_{i}, \gamma_{i}(i=1, \cdots 4)$ are the angles between the $i$ th wire and the $X, Y$ and $Z$ axis. The relation of $\cos \gamma_{1}=\sin a_{1}, \cos \gamma_{1}=\sin a_{1}$ can be obtained by the trigonometry. $l_{1}, l_{2}, l_{3}$ and $l_{4}$ are the length of wire $\mathrm{P}_{1} \mathrm{~A}, \mathrm{P}_{2} \mathrm{~B}$ and $\mathrm{P}_{3} \mathrm{C}$ respectively. $n$ is the distance from the platform to base of robot, and vary from $100 \mathrm{~mm}$ to $500 \mathrm{~mm}$.

The equation set (1) can be simplified as

$$
\left\{\begin{array}{l}
l_{1} \cos \alpha_{1}+c+l_{4} \cos \alpha_{4}=3 R / 2 \\
l_{1} \sin \alpha_{1}=l_{4} \sin \alpha_{4} \\
T_{1} \sin \alpha_{1}+T_{4} \sin \alpha_{4}=G \\
T_{1} \cos \alpha_{1}=T_{4} \cos \alpha_{4} \\
T_{1} \sin \alpha_{1} \cdot c=G \cdot d \\
l_{1} \sin \alpha_{1}+n=h
\end{array} .\right.
$$

Exam the equation set (2), for every specific height $n$, the only status of the wires is corresponded with only solution of $\alpha_{1}, \alpha_{4}, T_{1}, T_{4}, l_{1}$ and $l_{4}$. That is,

$$
\begin{aligned}
& \alpha_{1}=\arctan \frac{h-n}{\frac{c-d}{c}\left(\frac{3 R}{2}-c\right)}, \alpha_{4}=\arctan \frac{h-n}{\frac{d}{c}\left(\frac{3 R}{2}-c\right)}, \\
& l_{1}=\frac{h-n}{\sin \alpha_{1}}, l_{4}=\frac{h-n}{\sin \alpha_{4}}, T_{1}=\frac{G \cdot d}{\sin \alpha_{1} \cdot c}, T_{4}=\frac{G \cdot(c-d)}{\sin \alpha_{4} \cdot c}
\end{aligned} .
$$

Also, the length of wire $\mathrm{P}_{2} \mathrm{~B}$ and $\mathrm{P}_{3} \mathrm{C}$ can be obtained through $\mathrm{P}_{4} \mathrm{D}$, that is

$$
l_{2}=l_{3}=\sqrt{l_{4}^{2}+\frac{(\sqrt{3} R-b)^{2}}{4}} \text {. }
$$

The simulation results are shown in Fig. 4

The Fig. 5 show us the linear relation between the changes of platform's height $n$ and wire's length $l_{1}\left(l_{2}\right)$ when horizontal up-down motion, which can improve the stability of robot movement.
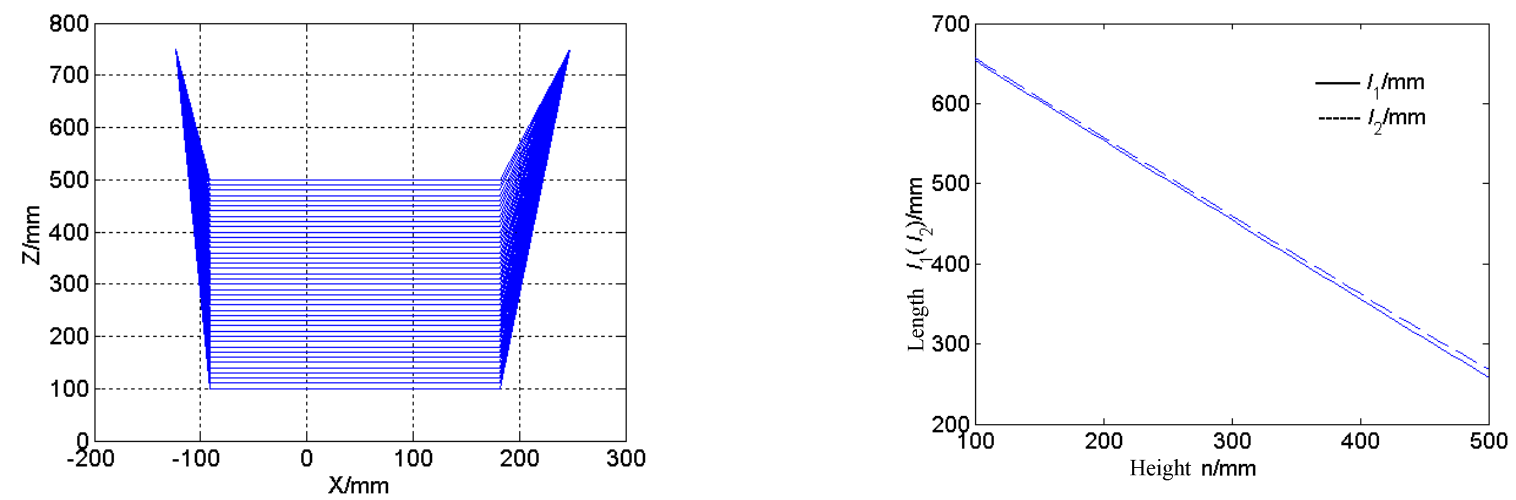

Fig. 4. The simulation of platform's horizontal Fig. 5. The relation between the changes of platform's up-down motion(side view) height $n$ and wire's length $l_{1}\left(l_{2}\right)$ when horizontal up-down motion 
Pitching motion. From Fig. 6, the kinematic equations of pitching motion can be written as

$$
\left\{\begin{array}{l}
l_{1} \cos \alpha_{1}+c \cos \theta+l_{4} \cos \alpha_{4}=3 R / 2 \\
l_{1} \sin \alpha_{1}=c \sin \theta+l_{4} \sin \alpha_{4} \\
l_{1} \sin \alpha_{1}+n-(c-d) \sin \theta-t \cos \theta=h \\
T_{1} \sin \alpha_{1}+T_{4} \sin \alpha_{4}=G \\
T_{1} \cos \alpha_{1}=T_{4} \cos \alpha_{4} \\
T_{1} \sin \alpha_{1} \cdot c \cdot \cos \theta+T_{1} \cos \alpha_{1} \cdot c \cdot \sin \theta \\
=G(d \cdot \cos \theta+t \sin \theta)
\end{array} .\right.
$$

The equation set (5) has 6 equations and 7 unknown variables $\alpha_{1}, \alpha_{4}, T_{1}, T_{4}, l_{1}, l_{4}$ and $\theta$. For every specific $n$ and $\theta$, the equation set (5) has unique solutions. To simplify the solving process, the special solving method is adopted here. By assuming several unknown variables as known and according the structure of robot and geometrical relationship, the other unknown variables can be expressed by the known. Then the solutions are judged by the given criteria. As shown in Fig.6, the extra variable $r$ and $s$ are introduced to identify the location of point $\mathrm{D}$. When assuming $\alpha_{1}$ and $l_{1}$ are known, the location of point A can be determined. Thus, we have

$$
\left\{\begin{array}{rl}
r & =\frac{3 R}{2}-l_{1} \cdot \cos \alpha_{1}-c \cdot \cos \theta \\
s & =l_{1} \cdot \sin \alpha_{1}-c \cdot \sin \theta \\
\alpha_{4} & =\arctan (s / r) \\
l_{4} & =\sqrt{s^{2}+r^{2}} \\
T_{4} & =G \cdot \cos \alpha_{1} /\left(\cos \alpha_{4} \sin \alpha_{1}+\sin \alpha_{4} \cos \alpha_{1}\right) \\
T_{1} & =T_{4} \cdot \cos \alpha_{4} / \cos \alpha_{1}
\end{array} .\right.
$$

Discriminant relation is

$$
\left|T_{1} \sin \alpha_{1} \cdot c \cdot \cos \theta+T_{1} \cos \alpha_{1} \cdot c \cdot \sin \theta-G(d \cos \theta+t \sin \theta)\right|<\varepsilon .
$$

where $\varepsilon$ is a small quantity.

Given the parameter $\alpha_{1} \in\left(0,90^{\circ}\right]\left(\right.$ step is $\left.0.01^{\circ}\right), l_{1}=300 \mathrm{~mm}, \theta \in\left[1^{\circ}, 30^{\circ}\right]$ (step is $\left.2^{\circ}\right)$. The simulation results are shown in Fig.8. And the relation between the changes of pitching angle $\theta$ and length $l_{2}$ in pitching motion, as shown in Fig. 7, shows the approximate linearity between $\theta$ and $l_{2}$.

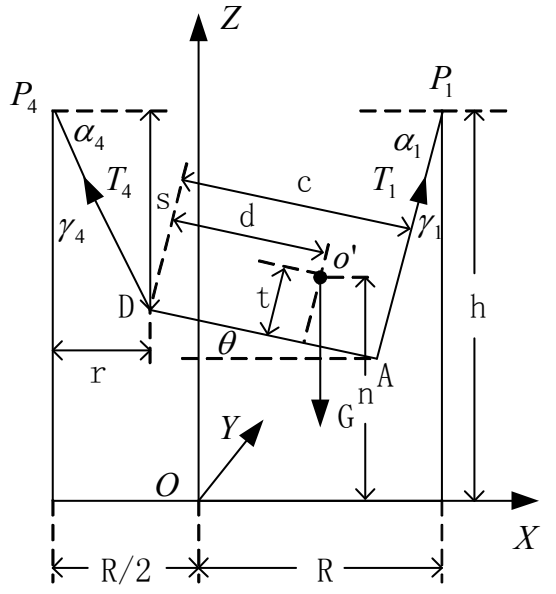

Fig. 6. Platform's pitching motion

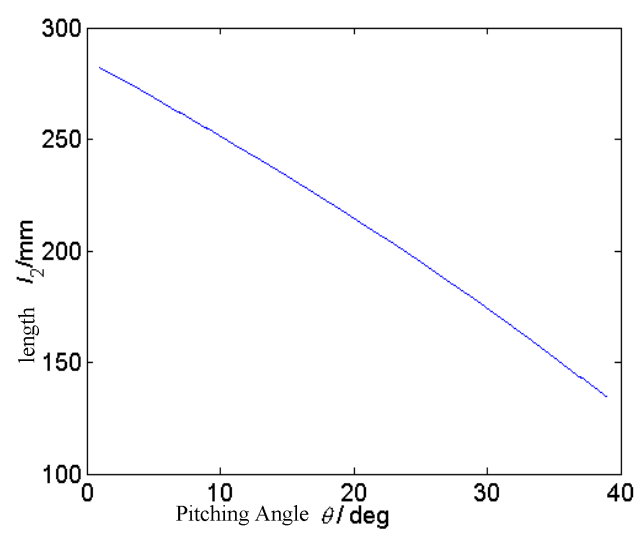

Fig. 7. Relation between the changes of pitching angle $\theta$ and $l_{2}$ in pitching motion 


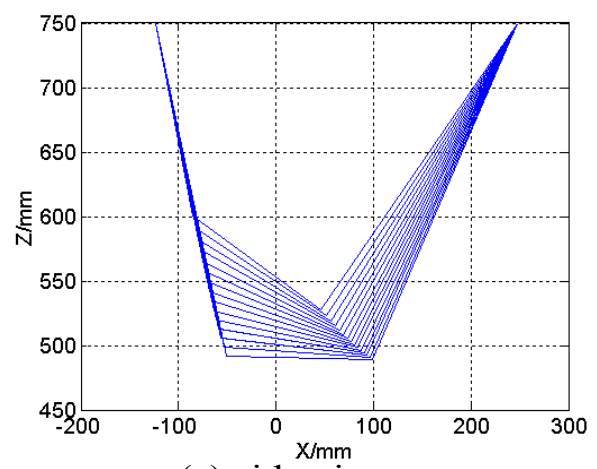

(a) side view

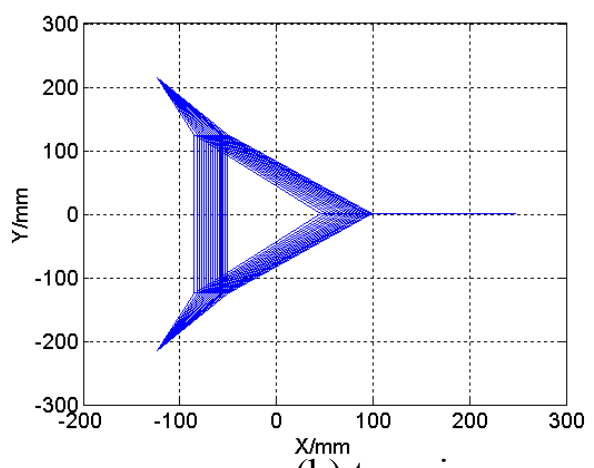

(b) top view

Fig. 8. The simulation of platform's pitching motion

Heeling motion. The heeling motion is shown in Fig. 9.

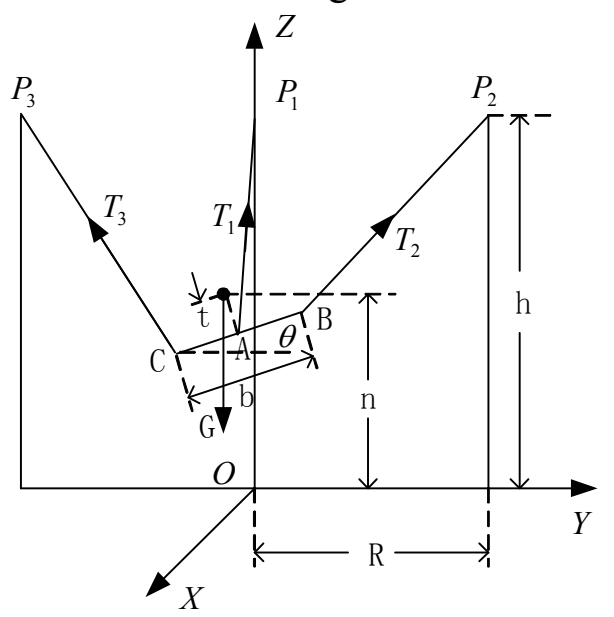

Fig. 9. Platform's heeling motion

The corresponding kinematic equation can be expressed as

$$
\left\{\begin{array}{l}
\cos ^{2} \alpha_{1}+\cos ^{2} \beta_{1}+\cos ^{2} \gamma_{1}=1 \\
\cos ^{2} \alpha_{2}+\cos ^{2} \beta_{2}+\cos ^{2} \gamma_{2}=1 \\
\cos ^{2} \alpha_{3}+\cos ^{2} \beta_{3}+\cos ^{2} \gamma_{3}=1 \\
T_{1} \cos \alpha_{1}+T_{2} \cos \alpha_{2}=T_{3} \cos \alpha_{3} \\
T_{3} \cos \beta_{3}+T_{2} \cos \beta_{2}=T_{1} \cos \beta_{1} \\
T_{1} \cos \gamma_{1}+T_{2} \cos \gamma_{2}+T_{3} \cos \gamma_{3}=G \\
-T_{3} \cos \gamma_{3} \cdot b \cdot \cos \theta-T_{3} \cos \alpha_{3} \cdot b \cdot \sin \theta-T_{1} \cos \gamma_{1} \cdot b \cdot \cos \theta / 2+ \\
T_{1} \cos \alpha_{1} \cdot b \cdot \sin \theta / 2+G(t \cdot \sin \theta+b \cdot \cos \theta / 2)=0 \\
-T_{1} \cos \gamma_{1} \cdot c-T_{1} \cos \beta_{1} \cdot b \cdot \sin \theta / 2+T_{3} \cos \beta_{3} \cdot b \cdot \sin \theta+G \cdot d=0 \\
T_{1} \cos \alpha_{1} \cdot c-T_{1} \cos \beta_{1} \cdot b \cdot \cos \theta / 2+T_{3} \cos \beta_{3} \cdot b \cdot \cos \theta=0 \\
l_{2} \cdot \cos \alpha_{2}+b \cdot \cos \theta+l_{3} \cdot \cos \alpha_{3}=\sqrt{3} R \\
l_{3} \cdot \cos \gamma_{3}=l_{2} \cdot \cos \gamma_{2}+b \cdot \sin \theta \\
l_{3} \cdot \cos \beta_{3}+c+l_{1} \cdot \cos \beta_{1}=3 R / 2 \\
l_{3} \cdot \cos \alpha_{3}+b \cos \theta / 2-l_{1} \cdot \cos \alpha_{1}=\sqrt{3} R / 2 \\
l_{3} \cdot \cos \gamma_{3}=l_{1} \cdot \cos \gamma_{1}+b \cdot \sin \theta / 2
\end{array}\right.
$$


where $\alpha_{1}, \alpha_{2}, \alpha_{3}, \beta_{1}, \beta_{2}, \beta_{3}, \gamma_{1}, \gamma_{2}, \gamma_{3}, T_{1}, T_{2}, T_{3}, l_{1}, l_{2}$ and $l_{3}$ are unknown variables, and heeling angle $\theta$ is known. For the convenience of solving, the functions related with $\alpha_{1}, \alpha_{2}, \alpha_{3}, \beta_{1}$, $\beta_{2}, \beta_{3}, \gamma_{1}, \gamma_{2}$ and $\gamma_{3}$, and expressed as cosine function, such as $\cos \alpha_{i}, \cos \beta_{i}$ and $\cos \gamma_{i}(i=1,2,3)$, can be viewed as a variable. Gauss-Newton method is used to solve the equations, and the simulation results are illustrated in Fig. 10.

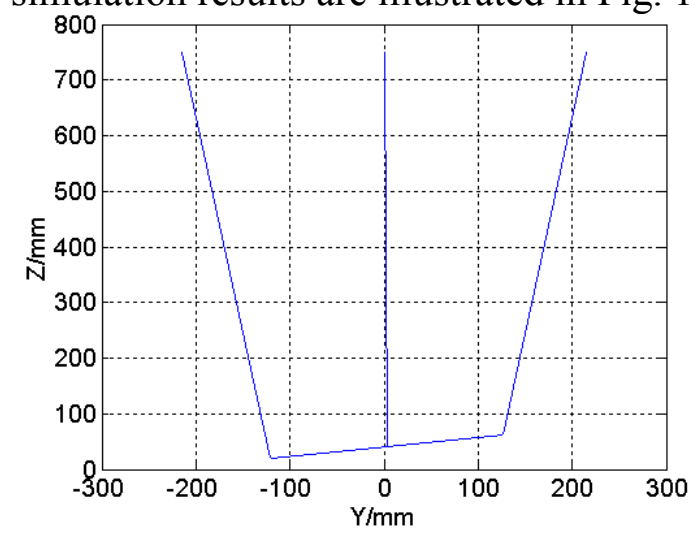

(a) front view

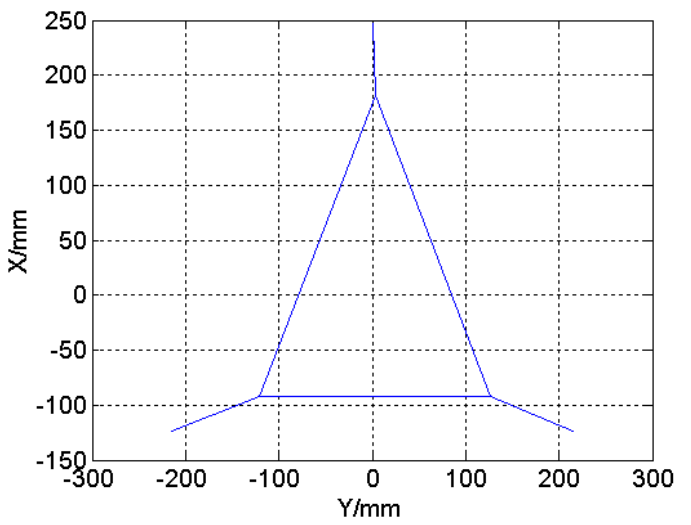

(b) top view

Fig. 10. The figure of platform's heeling motion $\left(\theta=10^{\circ}\right)$

From the heeling analysis, the center of platform drift $0.5 \mathrm{~mm} \sim 4.5 \mathrm{~mm}$ from center of the base by $\Delta=l_{1} \cdot \cos \beta_{1}$, when the heeling angle varies from $1^{\circ}$ to $20^{\circ}$. Reduce the size of the platform or increase the height the pillar, the scope of heeling can be enlarged.

\section{Summary}

In the paper, a novel new gravity-constrained (GC) three-wire-driven (TWD) parallel robot is proposed. With its mechanism model, three typical kinematic analytical models, including horizontal up-down motion, pitching motion and heeling motion, and their corresponding simulations are given in detail. The simulation results show the robot has good movement stability. The paper can provide useful materials to study of dynamics and control on wire-driven robot.

\section{Acknowledgements}

This work was financially supported by the Fundamental Research Fund for the Central Universities (N100503001).

\section{References}

[1] N. G. Dagalakis, J. S. Albus, B.-L. Wang, J. Unger, and J. D. Lee. Stiffness study of a parallel link robot crane for shipbuilding applications. Journal of Offshore Mechanics and Arctic Engineering, 1989, 111(3): 183-193.

[2] S. Tadokoro, S. Nishioka, T. Takamori, and K. Maeda. On fundamental design of wire Configurations of wire driven parallel manipulators with redundancy. Proceedings of Japan-U.S.A. Symposium on Flexible Automation, volume 1, 151-158, 1996.

[3] P.G. Clem, M. Rodriguez, J.A. Voigt and C.S. Ashley, U.S. Patent 6,231,666. (2001)

[4] Sadao Kawamura, Hitoshi Kino and Choe Won, High-speed manipulation by using parallel wire-driven robots[J] Robotica, 12000, 8(1):13-21.

[5] J. Albus, R. Bostelman, and N. Dagalakis. The nist robocrane. J. of Robotic Systems, 1993, 10(5):709-724.

[6] Maier T. Woernle C, Kinematic control of cable suspension robots 1997. 